\title{
'Asîr, eine Landschaft im südwestlichen Saudi-Arabien
}

Spricht man von Saudi-Arabien, denkt man gerne an Wuisten, Oel und unermesslichen Reichtum oder an Wiustenstämme, die mit ihren Kamelen die Weiten des Landes durchziehen. Das diesem Artikel beigegebene Bild entspricht diesen Vorstellungen kaum: Ein dunkel gekleideter Mann führt sein Kamel einer steinigen Piste entlang, im Hintergrund erhebt sich eine Häusergruppe, davor befinden sich einige grüne Felder. Die Aufnahme stammt aus 'Asîr, dem südwestlichen SaudiArabien. 'Asîr umfasst den an Jemen nördlich anschliessenden Abschnitt der jungen, stark gegliederten Ketten, die sich in Nord-Süd Richtung parallel zum Roten Meer erstrecken. Die Landschaft entspricht daher in manchem den westlichen Gebirgsregionen des Jemens viel mehr als dem trockenen zentralen und östlichen SaudiArabien. Dank Ausläufern des Südwestmonsuns und gelegentlichen Zyklonen, die im Winter von Norden her eindringen, ist in 'Asîr Regenfeldbau möglich (Niederschläge ca. $300-400 \mathrm{~mm} / \mathrm{Jahr}(1)$ ). Im ganzen übrigen Land hingegen ist ohne $\mathrm{Be}-$ wässerung kein Ackerbau denkbar. Die Niederschläge in 'Asîr fallen meist in Form von heftigen, kurzen Schauern. Auf den von der Vegetation kaum geschützten Flächen - wie z.B. der Hang im Bildhintergrund - versickert daher nur wenig Regenwasser. Sehr viel fliesst oberflächlich ab. Flussbette, welche die grösste Zeit des Jahres trocken liegen, verwandeln sich so in kürzester Zeit in reissende Ströme, die immer wieder zu Ueberschwemmungen führen und Menschenleben sowie wertvolles Kulturland vernichten.

Seitalters her versuchte der Mensch, diese Wasser für die Landwirtschaft zu nutzen. Kunstvolle Dämme wurden errichtet, um die dahinschiessenden Wassermassen aus den Flussbetten auf die benachbarten Felder zu leiten. Das wohl berühmteste Bauwerk dieser Art ist der Damm von Márib im Jemen, erbaut im 7.Jh.v.Chr. Nach mehreren Zerstörungen und Rekonstruktionen brach er.im 6.Jh.n.Chr. (?) erneut und wurde daraufhin endgiiltig aufgegeben. Es wird gegenwärtig untersucht, ob der Damm neu erstellt und der Bewässerung wieder nutzbar gemacht werden könnte. Anstelle von grossen Dämmen werden sehr oft kleine Erddämme errichtet, die aber nach jedem Hochwasser wieder neu gebaut werden müssen.

Auch die Ackerterrassen, die auf dem Bild sichtbar sind, dienen der Nutzbarmachung der Niederschläge. Diese können auf den praktisch horizontalen Terrassenflächen nicht oberflächlich abfliessen und erlauben damit eine gute Durchfeuchtung des Bodens. Nur das überschüssige Wasser wird mit speziellen Ueberläufen über die sorgfältig geschichteten Steinmauern auf die nächsttiefere Terrassenfläche geleitet. Trotz allen Vorkehrungen geschieht es allerdings immer wieder, dass solche Mauern bei heftigen Regen einstürzen und damit die Felder gefährden. Ständige Unterhalts- und Reparaturarbeiten sind nötig. Häufig werden nicht nur die Feldflächen, sondern auch ungenutzte Gebiete zur Wassernutzung einbezogen, indem dort Regenwasser gesammelt und den Feldern zugeleitet wird. Aus langer Erfahrung weiss man, wo Terrassen angelegt werden können, und wo es die lokalen Verhältnisse als "nicht mehr sinnvoll erscheinen lassen. So fällt auch auf dem Bild auf, dass der Hang über dem Dorf nicht terrassiert ist. Er dient als spärliche Weide für Schafe. Das alles zeigt, dass die Kunst des Terrassenbaus und der Nutzbarmachung der Niederschläge für die Landwirtschaft in 'Asîr, Jemen und den angrenzenden Regionen hoch entwickelt ist. Nur so ist es möglich, unter den herrschenden Reliefund $K l$ imabedingungen erfolgreich Regenfeldbau zu betreiben. Zusätzlich zu den beschriebenen Massnahmen wird wo immer möglich Grundwasser für die Bewässerung verwendet. Erfolgte ursprünglich das Heben des Wassers von Hand oder mit $\mathrm{Hilfe}$ von Tieren, werden heute mehr und mehr Pumpen eingesetzt. Sie erlauben es, grössere Flächen zu bewässern, sind aber in Ankauf und Betrieb relativ teuer und führen bei falschem Einsatz oft auch zur Uebernutzung der Grundwasservorräte.

Die Landnutzung hängt in 'Asîr stark von der Höhenlage und der lokalen orographischen Situation $a b(1)$. So sind Dattelpalmen und Bananen auf Höhen unter 1200 m.ü.M. beschränkt. Bis ca. 1700 m.ü.M. folgen neben Hirsearten, Mango und Papaya auch Kaffee und Qât (Catha edulis). Qât ist ein immergrüner Busch, dessen Blätter vor allem im Jemen häufig gekaut werden. Sie haben stimulierende Wirkung. In Saudi-Arabien ist der Qátanbau sehr streng kontrolliert, der Qátgenuss praktisch verboten. In Lagen über 1700 m.ü.M. finden sich dann Hirsearten, Mais, Weizen und Gerste, dazu v.a. auf bewässerten Feldern auch Gemuise und Futterpflanzen. Die auf dem Bild dargestellte Region befindet sich in der obersten der drei Höhenstufen. Hier erfolgt der An-

Dr.Hermann Escher, Altenbergstr. 20, 3013 Bern 
bau auch heute noch weitgehend von Hand und mit den traditionellen Geräten. Von Regierungsseite wird aber viel unternommen, um die Verwendung neuer Sorten und den Gebrauch von Dünger zu fördern und parallel dazu einen Beratungsdienst aufzubauen. Dabei ist allerdings zu bedenken, dass diesen Bemühungen enge Grenzen gesetzt sind: Eine Mechanisierung Z.B. wird wegen der kleinen Feldgrössen nur bedingt möglich sein.

Saudi-Arabien verfolgt weitgespannte Pläne bei der Erhöhung der landwirtschaftlichen Produktion. Weil die Möglichkeiten für Regenfeldbau landesweit betrachtet so beschränkt sind, basieren die Anstrengungen v.a. auf der künstlichen Bewässerung. Die Landwirtschaft ist heute bereits der grösste Wasserkonsument (2). Der Neuerschliessung von Wasserreserven kommt daher grosse Bedeutung zu. Einerseits werden neue Grundwasservorräte nutzbar gemacht, anderseits baut man Anlagen zur Entsalzung von Meerwasser. Die Arbeiten auf dem Landwirtschaftssektor beziehen sich sowohl auf die Erhöhung der Getreideproduktion als auch auf die Steigerung der Produktion von Fleisch, Eiern und Geflügel. Das Ziel Saudi-Arabiens ist es, den heute noch tiefen Grad der Selbstversorgung mit landwirtschaftlichen Gütern in den kommenden Jahren wesentlich zu erhöhen (2).

In neuester Zeit stellen sich der Landwirtschaft in 'Asîr ernsthafte Probleme, weil Arbeitskräfte fehlen (3). Die niedrigen landwirtschaftlichen Einkommen und die zunehmenden Möglichkeiten, in anderen Bereichen der Wirtschaft ein Auskommen zu finden, veranlassen immer mehr Leute, aus dieser Bergregion abzuwandern. Z.T. bleiben die Familien der Emigranten zurück und erhalten von ihnen regelmässig Geld zugeschickt, z.T. wandern sie ebenfalls aus. Zeichen dieser Entwicklung sind in 'Asîr da und dort schon sichtbar: aufgegebene Felder und zerfallene Häuser.

Auf dem Bild ist allerdings davon nichts zu erkennen. Die Felder sind bestellt, das Dorf noch intakt. Lehm- und Steinbauten fügen sich zu einer geschlossenen Siedlung zusammen. Einige der Gebäude sind weiss verputzt, andere in der Farbe des Baumaterials belassen. Diese heben sich dadurch kaum von ihrer Umgebung $a b$. Ihre oft eindrückliche Höhe und Grösse weisen auf den hohen Stand der lokalen Baukunst hin. Die Steinmetzarbeiten sind in der Regel von hoher Qualität. Das erstaunt nicht, wenn man an die oben beschriebenen Dammbauten und Terrassen denkt.

Ueber die Zugehörigkeit der Dörfer zu einzelnen Stammesgebieten kann vorläufig noch nichts ausgesagt werden, weil 'Asîr ethnologisch nur wenig bekannt ist. Ebenso sind die Strukturen der einzelnen Stämme und ihre gegenseitigen Beziehungen kaum erforscht. Der Mann im Vordergrund des Bildes trägt die in 'Asîr übliche Kleidung, zu der das Kopftuch und der Krummdolch (jambîya) gehören. Der Schirm dient sowohl als Sonnen- als auch als Regenschutz. Auch bei der Bevölkerung zeigen sich wieder die grossen Aehnlichkeiten zwischen 'Asîr und dem benachbarten Jemen. Gewisse Gebiete 'Asîrs gehörten denn auch zumindest zeitweise zum Jemen. Der Grenzverlauf blieb umstritten und wurde erst 1934 (Friede von al-Tو' if) festgelegt (4). Die republikanische Regierung des Jemens, die 1962 nach dem Sturz des letzten Imams an die Macht kam, anerkannte die Grenze gar erst 1970 auf saudi-arabischen Druck hin.

Bis vor wenigen Jahren blieben die Gebirgsregionen im südwestlichen Saudi-Arabien von Strassen praktisch unerschlossen. Kamele und Esel waren die einzigen Transportmittel. Nachdem nun aber die wichtigsten Orte des Landes durch ein Netz von gut ausgebauten Strassen miteinander verbunden sind, werden Strassen und Pisten (im Vordergrund des Bildes) auch in entlegene Gebiete erstellt. Autos übernehmen die Verbindungen mit den anderen Landesteilen, was den Güteraustausch natürlich erleichtert. Innerhalb der Region sind Kamel und Esel aber noch immer von Bedeutung. Nur wenige Bauern besitzen ein Auto, die Kosten für Autotransporte sind zudem relativ hoch. So begibt man sich z.B. noch immer meistens mit dem Kamel und nicht per Auto zu den Wochenmärkten.

Diese Wochenmärkte spielen in ländlichen Gebieten wie 'Asîr eine wichtige Rolle. Sie erlauben es der landwirtschaftlichen Bevölkerung, ihre Produkte abzusetzen und dafür andere für den täglichen Bedarf nötige Guiter zu kaufen. Das Warenangebot auf den Wochenmärkten ist äusserst vielfältig und umfasst heute neben lokal produzierten Gütern ein breites Spektrum von importierten Artikeln. Diese Importe lassen sich nicht nur aus dem Verkauf der landwirtschaftlichen Produktion finanzieren. Sehr viel Geld strömt dank den Ueberweisungen von Emigranten in die Region und wird zu Käufen auf den Märkten benutzt. Dadurch werden die Landwirtschaftsgebiete mehr und mehr von aussen abhängig. Aehnliche Entwicklungen zeigen sich auch im Jemen. So ermöglicht das Bild, das diesem Artikel zugrunde liegt, einen Einblick in die Gebirgsregionen des südwestlichen Saudi-Arabiens. Sie liegen etwas abseits der gegenwärtigen Brennpunkte der Wirtschaft und sind von den stürmischen Veränderungen der letzten Jahre nur am Rande erfasst worden. Um so mehr lassen sie die grossen Unterschiede erahnen zwischen den Landwirtschaftsgebieten und den wichtigen 0elzentren.

\section{Literatur}

1) Blume, Helmuth (Hrsg.): Saudi-Arabien, Erdmann Verlag Tübingen und Basel, 1976

2) Arab-Swiss Chamber of Commerce: Bulletin No. 22. Genf, Sept. 1977

3) Ministry of Information, The Kingdom of SaudiArabia: Outline of the Second Five Year Development Plan (ohne Jahr)

4) Wenner, M. Modern Yemen. The Johns Hopkins Press, 1967.

Foto aus dem Bildband: Walter Weiss, SAUDIARABIEN. D 224 Seiten, 114 Farbbilder, 1 doppelseitige Übersichtskarte. 7 Kartenskizzen, 4 Zeichnungen. Format $24 \times 30 \mathrm{~cm}$. 88.- . (C) Kümmerly+Frey, Geographischer Verlag, Bern. 


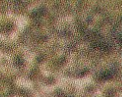

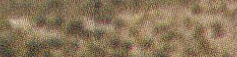

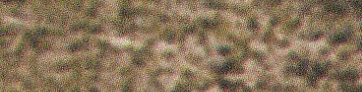

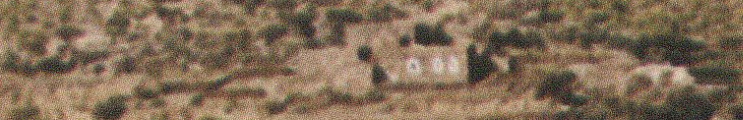

$3 x^{2}+2$

$+3$

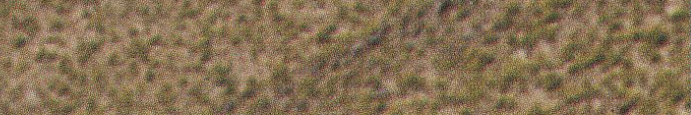

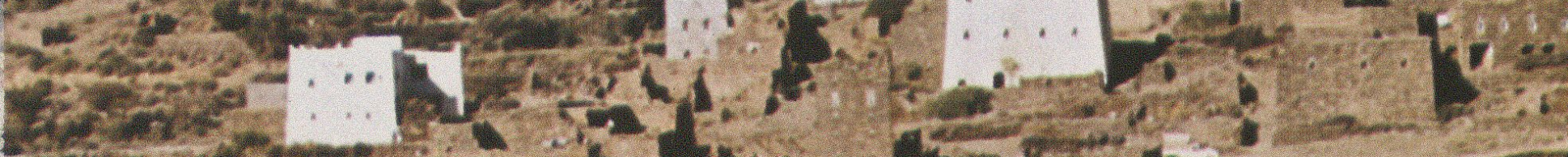

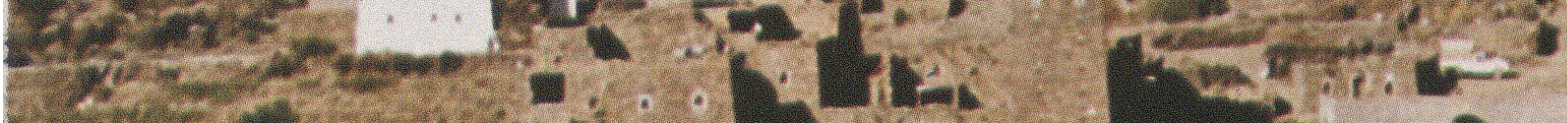

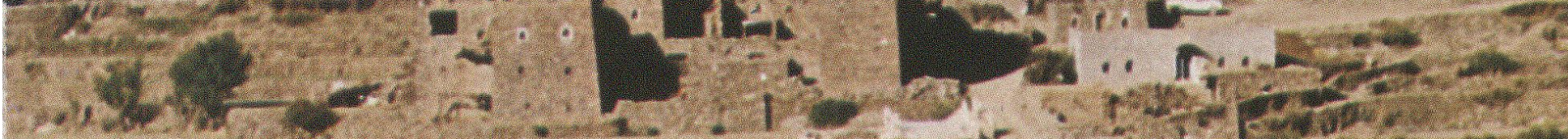

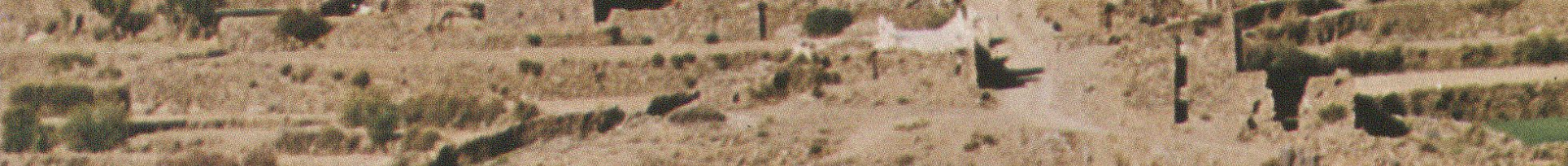
: at.

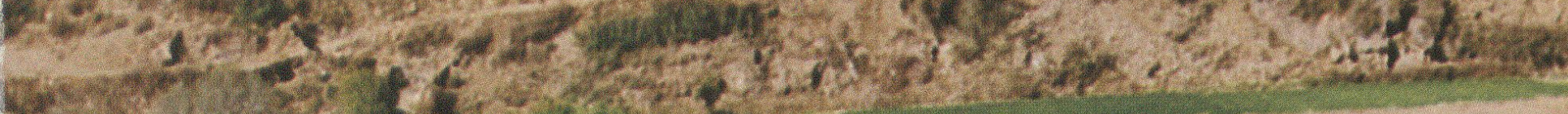

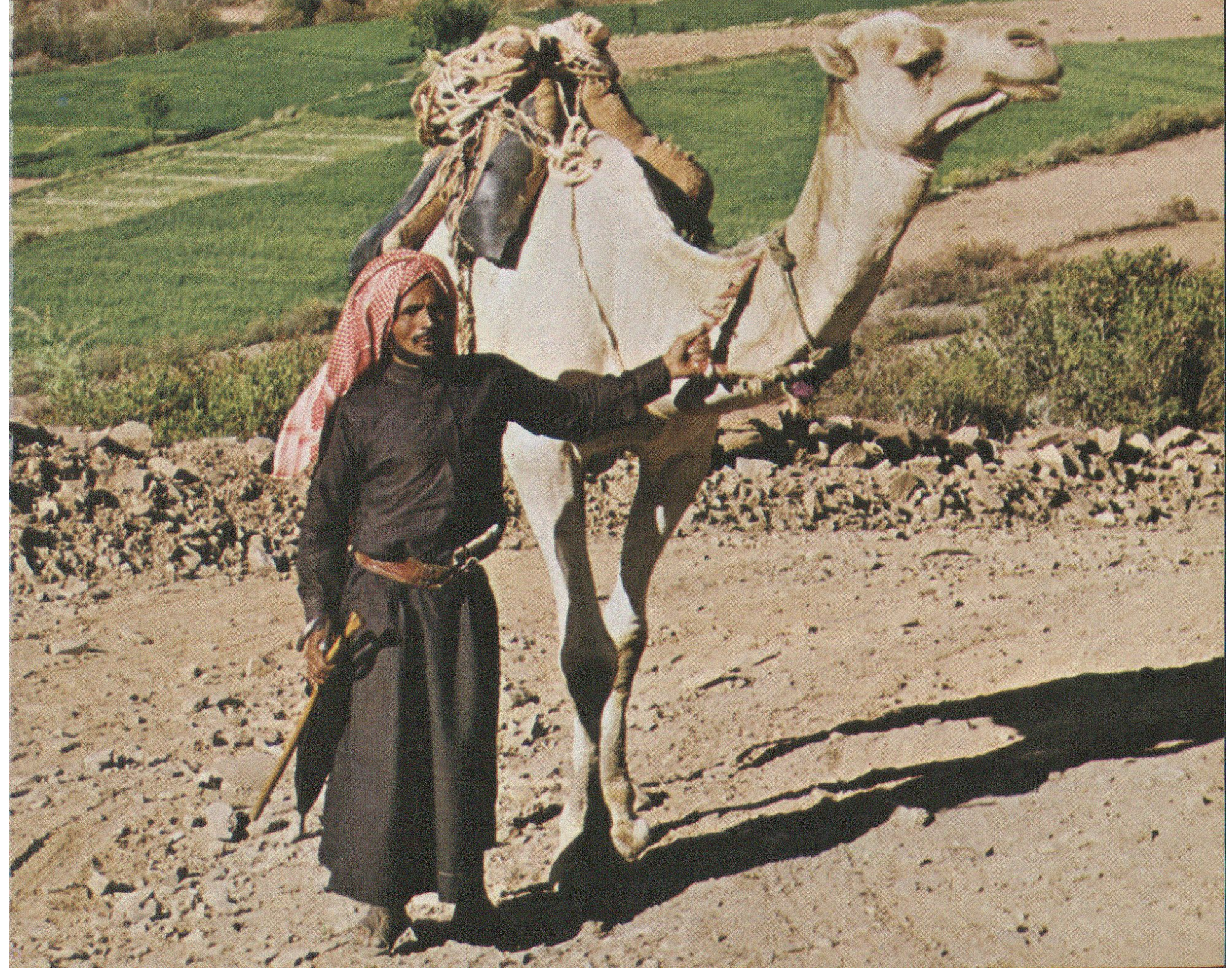

